Supplementary information

\title{
New Insight into Plasmid-Driven T7 RNA Polymerase (PDT7) \\ in Escherichia coli and Used as Genetic Amplifier for
}

\section{Biosensor}

\author{
Shih-I Tan, I-Son Ng* \\ Department of Chemical Engineering, National Cheng Kung University, \\ Tainan, Taiwan, 70101
}

*Corresponding author: I-Son $\mathrm{Ng}$

E-mail: yswu@mail.ncku.edu.tw

ORCID: 0000-0003-1659-5814

Tel: +866-62757575; Fax: +86-62344496 
A

Correct T7 promoter:

G A T A A T ACGACTCACTATAGGGAGAGG

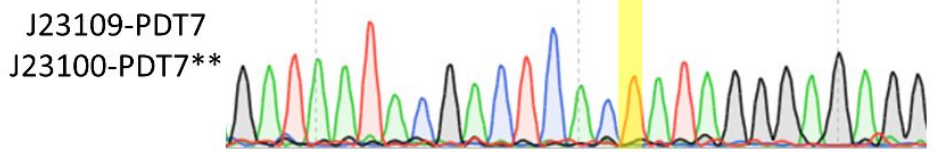

Mutated T7 promoter:

G A T A A T A C G A C T C A C ATA G G G A G A G G

J23114-PDT7*

J23105-PDT7*

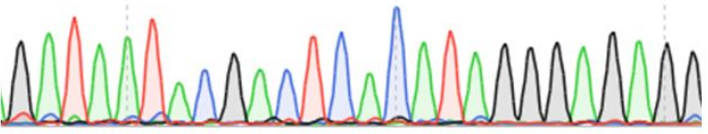

B

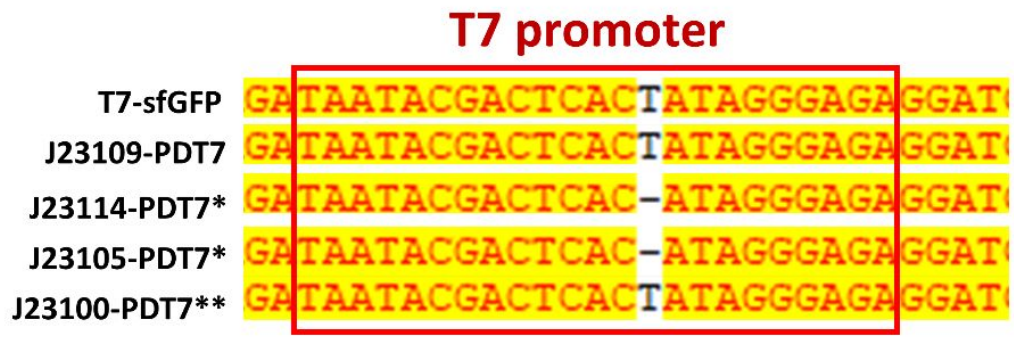

C

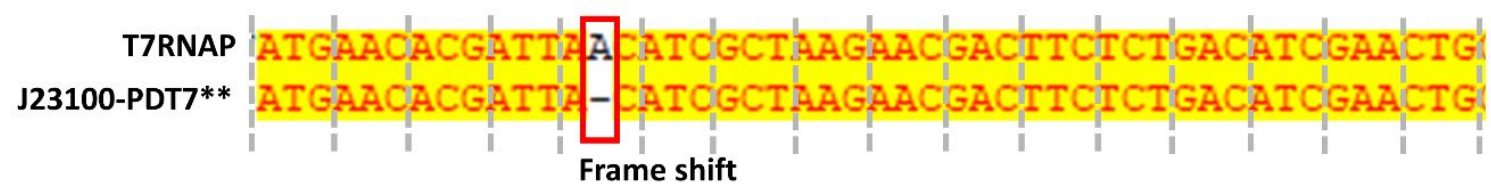

Figure S1. Sequence results of our constructed constitutive PDT7. (A) Chromatography figure of the sequence of $\mathrm{T} 7$ promoter region in our constructed constitutive PDT7. (B) Alignment of the sequencing result around the $\mathrm{T} 7$ promoter region. A single $\mathrm{T}$ missing at $14^{\text {th }}$ nucleotide is observed in the constructed pSU-J23114-PDT7* and pSU-J23105-PDT7* (C) Alignment of the sequencing results of T7RNAP in the constructed pSU-J23100-PDT7**. The missing nucleotide " $\mathrm{A}$ " is observed and resulted in the frame shift. 

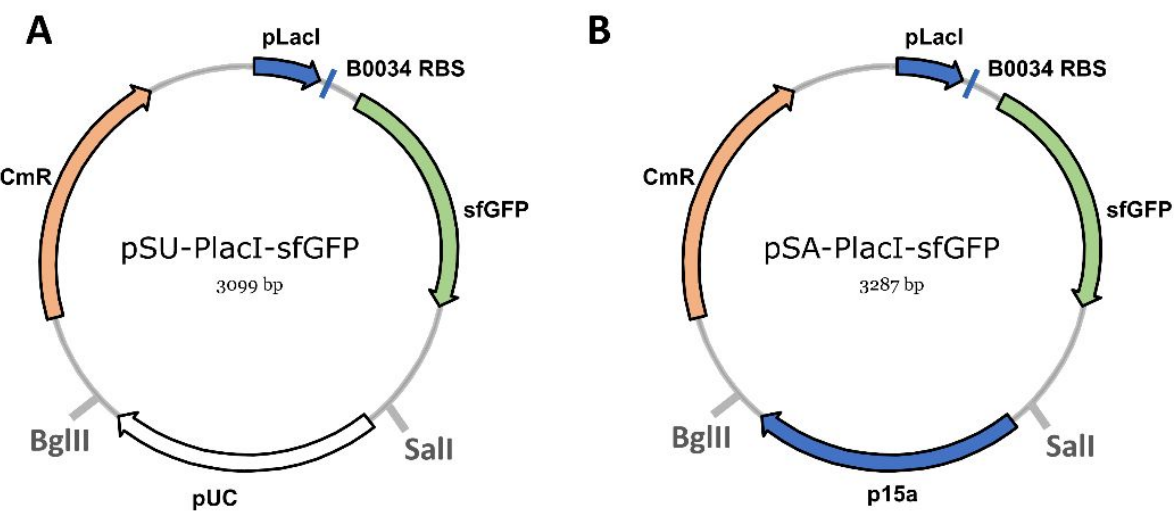

C
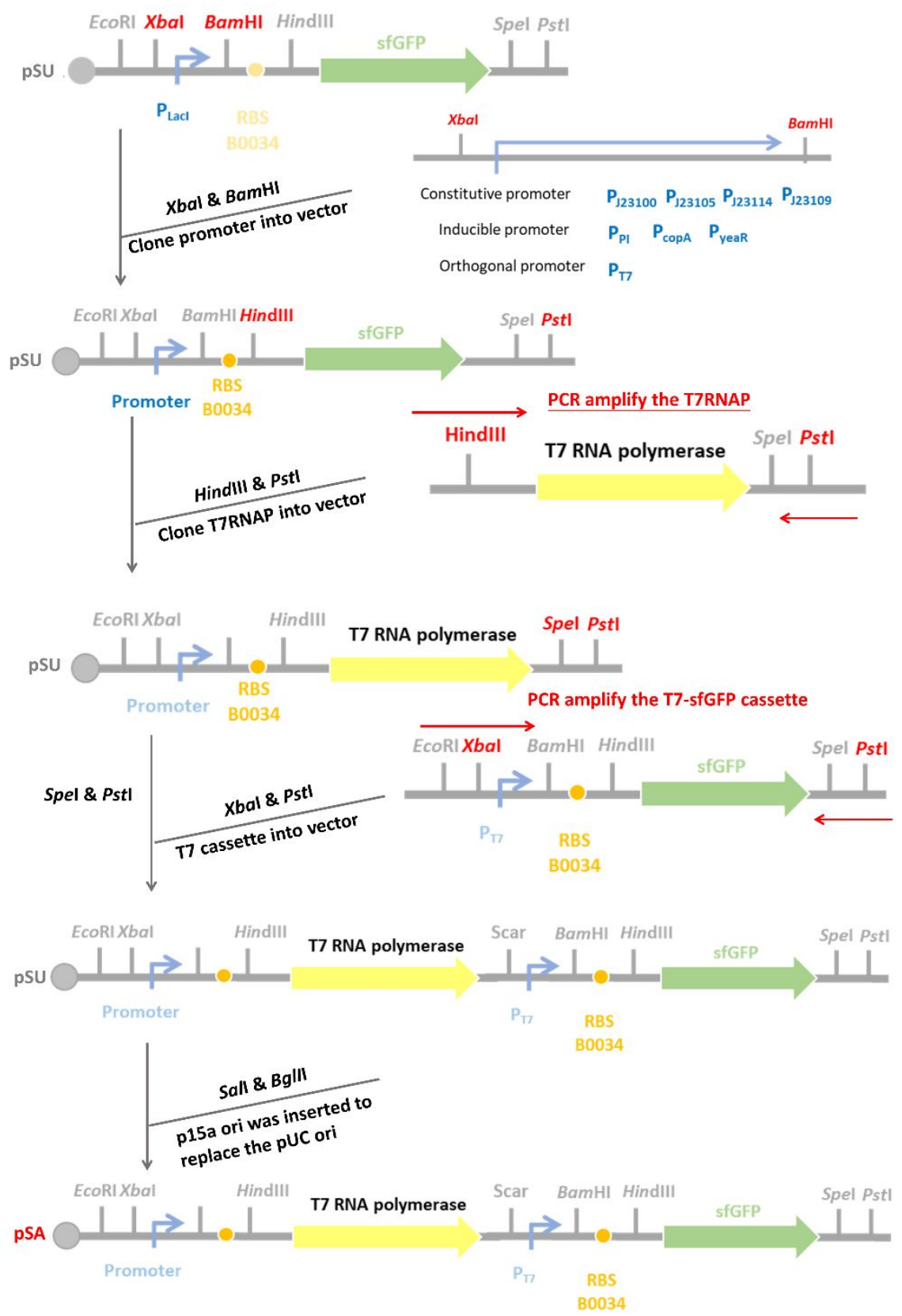

Figure S2. Flowchart of construction of plasmids. (A) Map of pSU-PLacI-sfGFP, (B) Map of pSA-PlacI-sfGFP, (C) Construction procedure used in this study. Red color indicated the utilized restriction sites. 

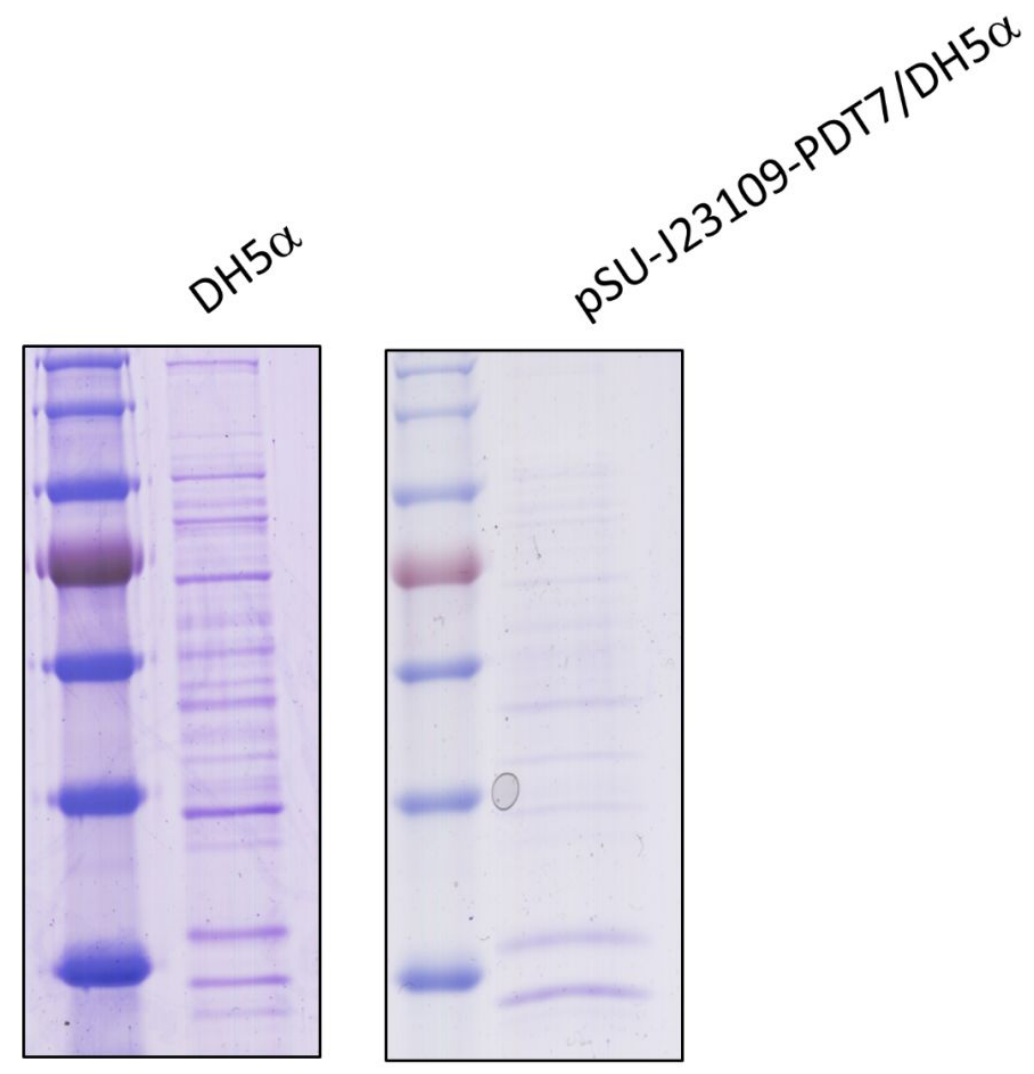

Figure S3. SDS-PAGE analysis of E. coli DH5 $\alpha$ (left), and DH5 $\alpha$ harboring pSU-J23109-PDT7 (right) for culturing at $8 \mathrm{~h}$. 
$\rightarrow$ sfGFP: $27.1 \mathrm{kDa}$
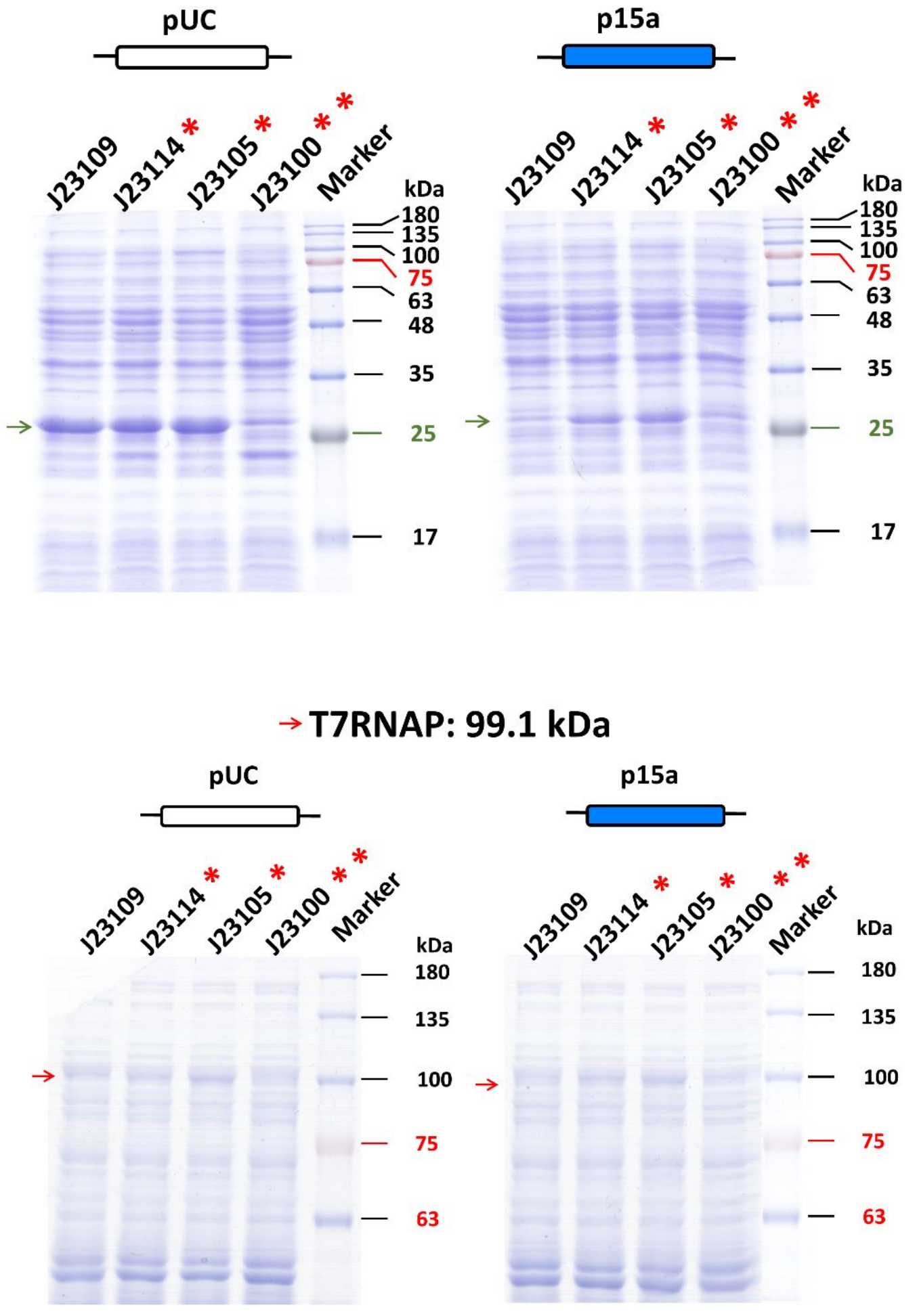

Figure S4. SDS-PAGE analysis of DH5 $\alpha$ harboring PDT7 genetic circuits. The green and red arrow indicates the targeted protein of sfGFP and T7RNAP, respectively. * indicates the mutation of the T7 promoter and ${ }^{* *}$ represented the frame shift on the T7RNAP. 


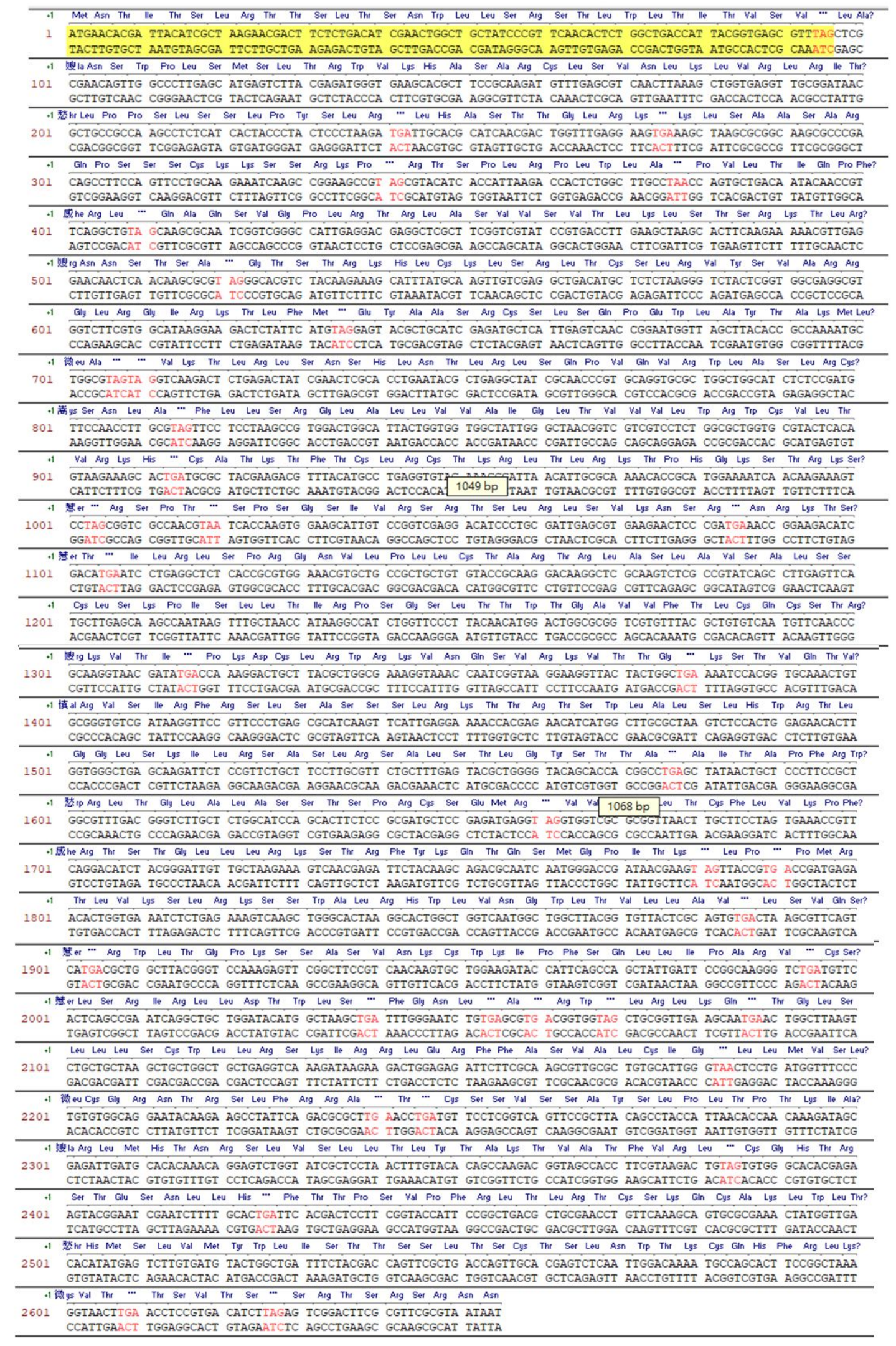

Figure S5. Translational analysis of mutated T7RNAP in pSU-J23100-PDT7**. The yellow color showed the first peptide that would be produced theoretically. The red color indicated the stop codon which caused from the frame shift. 
A B

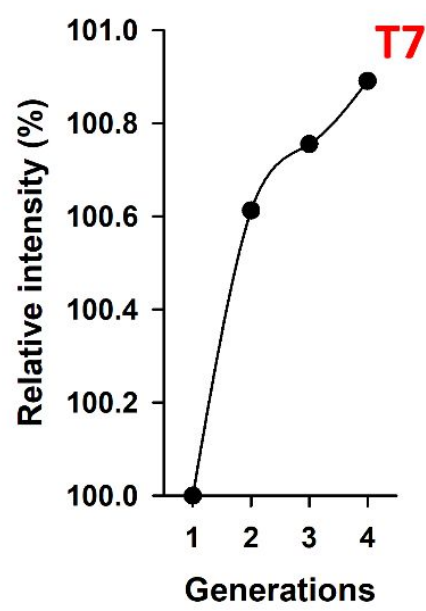

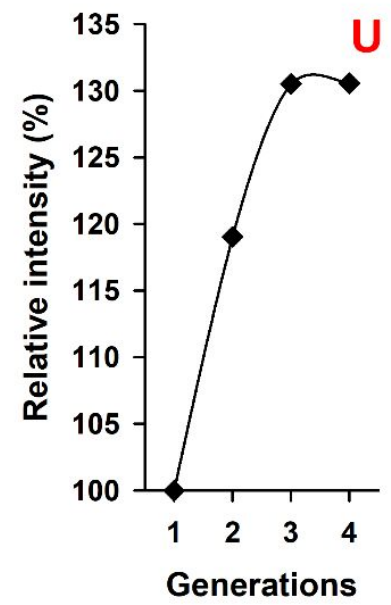

C

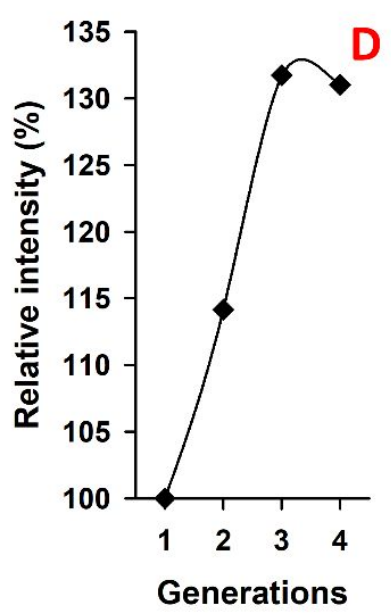

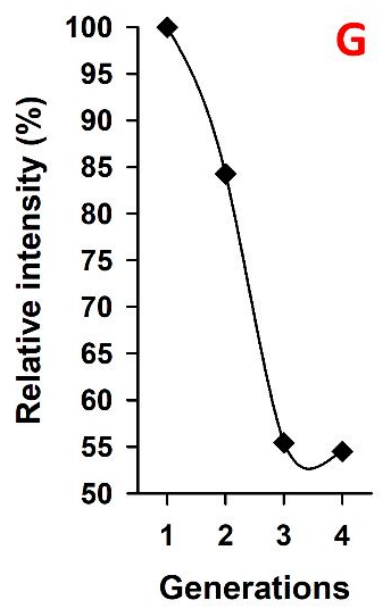

Figure S6. Gel analysis for band intensity of different proteins of T7, U, D and G. Relative intensity was used to illustrate the difference along with the generations for (A) T7, (B) U, (C) D and (D) G bands. 
A Carbonic anhydrase (CA)

$$
\mathrm{CO}_{2}+\mathrm{H}_{2} \mathrm{O} \stackrel{\mathrm{HpCA}}{\longleftrightarrow} \mathrm{HCO}_{3}^{-}+\mathrm{H}^{+}
$$

B Lysine decarboxylase (CadA)

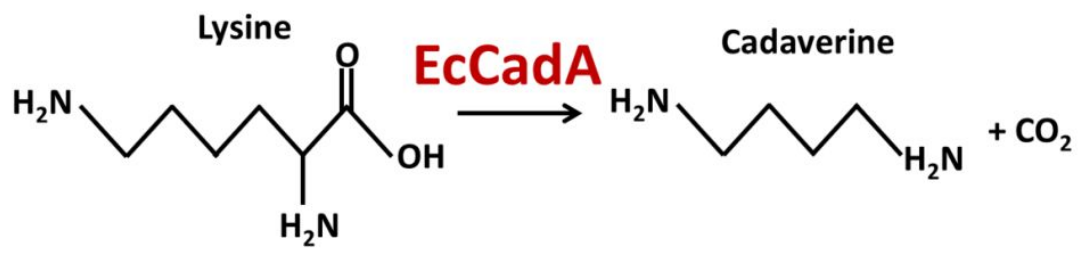

C Aminolevulinic acid synthase (HemA)

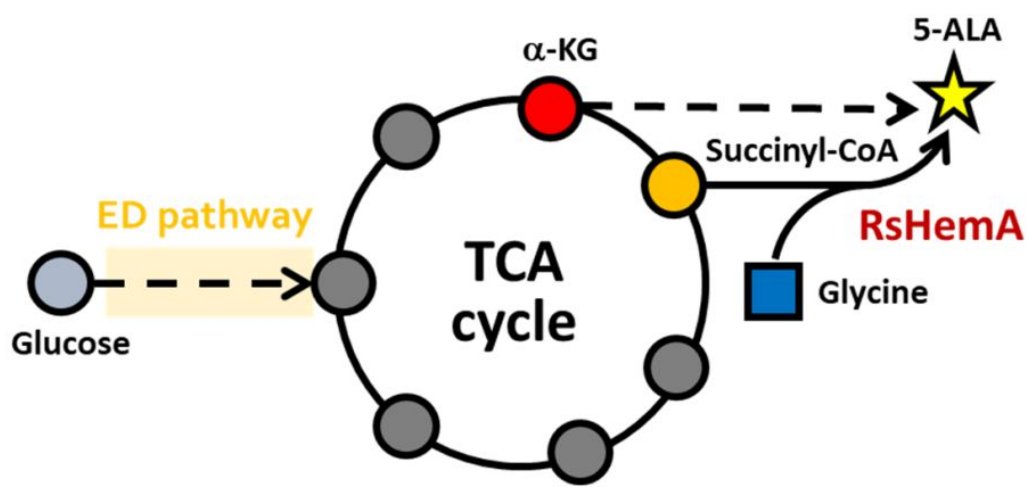

Figure S7. Illustration of enzymatic reaction of selected enzyme (A) Hydration of carbon dioxide catalyzed by carbonic anhydrase from the Helicobacter pylori. (B) Decarboxylation reaction of lysine to diaminopentene catalyzed by the lysine decarboxylase from E. coli. (C) Pathway evolving the 5-aminolevulinic acid production. The 5-ALA synthetase from the Rhodobacter sphaeroides was selected to produce the 5-ALA. 
Table S1 Constitutive promoter and the related mRNA expression level from iGEM.

\begin{tabular}{|c|c|c|}
\hline $\begin{array}{c}\text { Promoter } \\
\text { Bio-brick }\end{array}$ & Sequence (5' -3' $\left.^{\prime}\right)$ & $\begin{array}{c}\text { mRFP expression } \\
\text { (a.u. })\end{array}$ \\
\hline J23109 & TTTACAGCTAGCTCAGTCCTAGGGACTGTGCTAGC & 106 \\
\hline J23114 & TTTATGGCTAGCTCAGTCCTAGGTACAATGCTAGC & 256 \\
\hline J23105 & TTTACGGCTAGCTCAGTCCTAGGTACTATGCTAGC & 623 \\
\hline J23100 & TTGACGGCTAGCTCAGTCCTAGGTACAGTGCTAGC & 2547 \\
\hline
\end{tabular}

Table S2 Summary of sequencing results and cell growth in pSU-J231XX-PDT7 and pSU-T7-sfGFP

\begin{tabular}{|l|c|c|c|c|}
\hline Plasmid & T7 promoter & $\begin{array}{c}\text { J231XX } \\
\text { promoter }\end{array}$ & T7RNAP CDS & Average Growth Rate $^{\#}$ \\
\hline pSU-T7-sfGFP & $\mathrm{V}$ & N.D. & N.D. & 0.144 \\
\hline pSU-J23109-PDT7 & $\mathrm{V}$ & $\mathrm{V}$ & $\mathrm{V}$ & 0.050 \\
\hline pSU-J23114-PDT7* & Single T missing & $\mathrm{V}$ & $\mathrm{V}$ & 0.113 \\
\hline pSU-J23105-PDT7* & Single T missing & $\mathrm{V}$ & $\mathrm{V}$ & 0.110 \\
\hline pSU-J23100-PDT7** & $\mathrm{V}$ & $\mathrm{V}$ & Frame shift & 0.138 \\
\hline
\end{tabular}

\#The average cell growth rate was calculated as the following formula: OD600@12hr/12 (OD/hr)

Table S3 Effect of casamino acid addition in the instable expression of PDT7 at $4^{\text {th }}$ generation.

\begin{tabular}{|l|c|c|c|c|}
\hline \multirow{2}{*}{ Descriptions } & \multicolumn{3}{|c|}{ Addition of casamino acid (g/L) } \\
\cline { 2 - 5 } & 0 & 1 & 5 & 10 \\
\hline Relative specific fluorescence (\%) & 40 & 44 & 49 & 53 \\
\hline $\begin{array}{l}\text { Enhancement compared to the condition } \\
\text { without casamino acid (\%) }\end{array}$ & 0 & 4 & 9 & 13 \\
\hline $\begin{array}{l}\text { Specific enhancement on casamino acid } \\
\text { concentration (\%/g/L) }\end{array}$ & N.D. & 4 & 1.8 & 1.3 \\
\hline
\end{tabular}

Table S4 The information of inducible promoter used in this study.

\begin{tabular}{|l|l|l|l|l|}
\hline $\begin{array}{l}\text { Sensing } \\
\text { molecule }\end{array}$ & Promoter & $\begin{array}{l}\text { Organism } \\
\text { from }\end{array}$ & Sequence $\left(5^{\prime}-3^{\prime}\right)$ & Application \\
\hline Glucose & $\mathrm{P}_{\mathrm{PI}}$ & E. coli & $\begin{array}{l}\text { TTGACAGCTAGCTCAAATGTGATTATAATC } \\
\text { ACATTT }\end{array}$ & $\begin{array}{l}\text { Diagnosis of urine } \\
\text { glucose for diabetic } \\
\text { prevention }\end{array}$ \\
\hline $\begin{array}{l}\text { Copper } \\
\text { ion }\end{array}$ & $\mathrm{P}_{\text {copA }}$ & E. coli & $\begin{array}{l}\text { GCCGGATGCGGCGTAAACGCCTTATCCGGC } \\
\text { CTACGGTTCAGGCACAGGTTTGTAGGCCTG } \\
\text { ATAAGACGCGTAAGCGTCGCATCAGGCAAT } \\
\text { GGTGCTCGGATGCCGGATGCGGCGTAAACG } \\
\text { CCTTATCCGGCCTACGGTTCAGGCACAAGT } \\
\text { TTGTAGGCCTGATAAGACGGGTAAGCGTCG } \\
\text { CATCAGGCAATGGTGCTCGGATGCCGGATG } \\
\text { CGGCGTGAACGCCTTATCCGGCCTAC }\end{array}$ & \\
\hline
\end{tabular}


Table S5 Strains, plasmids and primers used in this study.

\begin{tabular}{|c|c|c|}
\hline Name & Description & Remark \\
\hline \multicolumn{3}{|l|}{ Strains } \\
\hline E. coli $\mathrm{DH} 5 \mathrm{a}$ & $\begin{array}{l}\text { F-, } \Delta(\operatorname{argF}-\mathrm{lac}) 169, \varphi 80 \text { dlacZ58(M15), } \Delta \text { phoA8, } \\
\text { glnX44(AS), } \lambda-\text {, deoR481, rfbC1, gyrA96(NalR), recA1, } \\
\text { endA1, thiE1, hsdR17 }\end{array}$ & $\begin{array}{l}\text { Used for } \\
\text { molecular } \\
\text { cloning }\end{array}$ \\
\hline \multicolumn{3}{|c|}{ ( } \\
\hline $\begin{array}{l}\text { pSB1C3-P }{ }_{\mathrm{BAD}^{-}} \text {-T7RNAP-TT } \\
\left(\mathrm{BBa} \_1450004\right)\end{array}$ & $\begin{array}{l}5018 \mathrm{bp}, \mathrm{Cm}^{\mathrm{R}} \text {, pUC ori, BAD promoter, B0034 RBS, } \\
\text { T7RNAP, B0010/B0012 double terminator }\end{array}$ & $\begin{array}{l}\text { iGEM, } \\
\text { amplification } \\
\text { of T7RNAP }\end{array}$ \\
\hline pSU-PT7-sfGFP & 2922 bp, $\mathrm{Cm}^{\mathrm{R}}$, pUC ori, T7 promoter, B0034 RBS, sfGFP & This study \\
\hline pSU-J23100-sfGFP & 2934 bp, $\mathrm{Cm}^{\mathrm{R}}$, pUC ori, J23100 promoter, B0034 RBS, sfGFP & This study \\
\hline pSU-J23105-sfGFP & 2934 bp, $\mathrm{Cm}^{\mathrm{R}}$, pUC ori, J23105 promoter, B0034 RBS, sfGFP & This study \\
\hline pSU-J23114-sfGFP & 2934 bp, $\mathrm{Cm}^{\mathrm{R}}, \mathrm{pUC}$ ori, J23114 promoter, B0034 RBS, sfGFP & This study \\
\hline pSU-J23109-sfGFP & 2934 bp, $\mathrm{Cm}^{\mathrm{R}}$, pUC ori, J23109 promoter, B0034 RBS, sfGFP & This study \\
\hline pSA-J23100-sfGFP & $3122 \mathrm{bp}, \mathrm{Cm}^{\mathrm{R}}$, p15a ori, J23100 promoter, B0034 RBS, sfGFP & This study \\
\hline pSA-J23105-sfGFP & $3122 \mathrm{bp}, \mathrm{Cm}^{\mathrm{R}}, \mathrm{p} 15 \mathrm{a}$ ori, J23105 promoter, B0034 RBS, sfGFP & This study \\
\hline pSA-J23114-sfGFP & 3122 bp, $\mathrm{Cm}^{\mathrm{R}}, \mathrm{p} 15 \mathrm{a}$ ori, J23114 promoter, B0034 RBS, sfGFP & This study \\
\hline pSA-J23109-sfGFP & 3122 bp, $\mathrm{Cm}^{\mathrm{R}}, \mathrm{p} 15 \mathrm{a}$ ori, J23109 promoter, B0034 RBS, sfGFP & This study \\
\hline pSU-J23100-PDT7** & $\begin{array}{l}5780 \mathrm{bp}, \mathrm{Cm}^{\mathrm{R}} \text {, pUC ori, J23100 promoter, T7 promoter, B0034 RBS, } \\
\text { T7RNAP**(frame shift), B0010/B0012 double terminator, sfGFP }\end{array}$ & This study \\
\hline pSU-J23105-PDT7* & $\begin{array}{l}5780 \text { bp, } \mathrm{Cm}^{\mathrm{R}} \text {, pUC ori, J23105 promoter, mutated T7 promoter, } \\
\text { B0034 RBS, T7RNAP, B0010/B0012 double terminator, sfGFP }\end{array}$ & This study \\
\hline pSU-J23114-PDT7* & $\begin{array}{l}5780 \mathrm{bp}, \mathrm{Cm}^{\mathrm{R}} \text {, pUC ori, J23114 promoter, mutated T7 promoter, } \\
\text { B0034 RBS, T7RNAP, B0010/B0012 double terminator, sfGFP }\end{array}$ & This study \\
\hline pSU-J23109-PDT7 & $\begin{array}{l}5780 \mathrm{bp}, \mathrm{Cm}^{\mathrm{R}} \text {, pUC ori, J23109 promoter, T7 promoter, B0034 RBS, } \\
\text { T7RNAP, B0010/B0012 double terminator, sfGFP }\end{array}$ & This study \\
\hline pSA-J23100-PDT7** & $\begin{array}{l}5968 \text { bp, } \mathrm{Cm}^{\mathrm{R}} \text {, p15a ori, J23100 promoter, T7 promoter, B0034 RBS, } \\
\text { T7RNAP **(frame shift), B0010/B0012 double terminator, sfGFP }\end{array}$ & This study \\
\hline pSA-J23105-PDT7* & $\begin{array}{l}5968 \mathrm{bp}, \mathrm{Cm}^{\mathrm{R}} \text {, p15a ori, J23105 promoter, T7 promoter, B0034 RBS, } \\
\text { T7RNAP, B0010/B0012 double terminator, sfGFP }\end{array}$ & This study \\
\hline pSA-J23114-PDT7* & $\begin{array}{l}5968 \text { bp, } \mathrm{Cm}^{\mathrm{R}} \text {, p15a ori, J23114 promoter, mutated T7 promoter, } \\
\text { B0034 RBS, T7RNAP, B0010/B0012 double terminator, sfGFP }\end{array}$ & This study \\
\hline pSA-J23109-PDT7 & $\begin{array}{l}5968 \text { bp, } \mathrm{Cm}^{\mathrm{R}} \text {, p15a ori, J23109 promoter, mutated T7 promoter, } \\
\text { B0034 RBS, T7RNAP, B0010/B0012 double terminator, sfGFP }\end{array}$ & This study \\
\hline pSU-J23114-PDT7-HpCA* & $\begin{array}{l}5812 \text { bp, } \mathrm{Cm}^{\mathrm{R}} \text {, pUC ori, J23114 promoter, mutated T7 promoter, } \\
\text { B0034 RBS, T7RNAP, B0010/B0012 double terminator, HpCA }\end{array}$ & This study \\
\hline pSU-J23114-PDT7-EcCadA* & $\begin{array}{l}7205 \text { bp, } \mathrm{Cm}^{\mathrm{R}} \text {, pUC ori, J23114 promoter, mutated T7 promoter, } \\
\text { B0034 RBS, T7RNAP, B0010/B0012 double terminator, EcCadA }\end{array}$ & This study \\
\hline pSU-J23114-PDT7-RsHemA* & $\begin{array}{l}6284 \mathrm{bp}, \mathrm{Cm}^{\mathrm{R}} \text {, pUC ori, J23114 promoter, mutated T7 promoter, } \\
\text { B0034 RBS, T7RNAP, B0010/B0012 double terminator, RsHemA }\end{array}$ & This study \\
\hline
\end{tabular}




\begin{tabular}{|c|c|c|}
\hline \multicolumn{3}{|l|}{ Primers } \\
\hline XbaI-PT7-F & $\underline{\text { CTAGATAATACGACTCACTATAGGGAGAG }}$ & This study \\
\hline BamHI-PT7-R & GATCCTCTCCCTATAGTGAGTCGTATTAT & This study \\
\hline XbaI-PJ23105-F & CTAGATTTACGGCTAGCTCAGTCCTAGGTACTATGCTAGCE & This study \\
\hline BamHI-PJ23105-R & $\begin{array}{l}\underline{\text { GATCCGCTAGCATAGTACCTAGGACTGAGCTAGCCGTAAA }} \\
\underline{\mathrm{T}}\end{array}$ & This study \\
\hline XbaI-PJ23114-F & CTAGATTTACAGCTAGCTCAGTCCTAGGGACTGTGCTAGC $\underline{\underline{G}}$ & This study \\
\hline BamHI-PJ23114-R & $\begin{array}{l}\underline{\text { GATCCGCTAGCACAGTCCCTAGGACTGAGCTAGCTGTAAA }} \\
\underline{\mathrm{T}}\end{array}$ & This study \\
\hline XbaI-PJ23109-F & CTAGATTTACAGCTAGCTCAGTCCTAGGGACTGTGCTAGC白 & This study \\
\hline BamHI-PJ23109-R & $\begin{array}{l}\underline{\text { GATCCGCTAGCACAGTCCCTAGGACTGAGCTAGCTGTAAA }} \\
\underline{\mathrm{T}}\end{array}$ & This study \\
\hline HindIII-T7RNAP-F & CGAAGCTTATGAACACGATTAACATCGCTAAGAACGAC & This study \\
\hline SpeI-Terminator-T7RNAP-R & CTACTAGTATATAAACGCAGAAAGGCCCACC & This study \\
\hline BglII-p15a-F & GCAGATCTTATTTATTCGGCGCAAAGTGCGT & This study \\
\hline SalI-p15a-R & GTGTCGACTACATTTAGGCGCTGCCATCTTG & This study \\
\hline E-X-PT7-sfGFP-F & $\begin{array}{l}\text { GAGAATTCGCGGCCGCTTCTAGATAATACGACTCACTATAG } \\
\text { GGAGAGG }\end{array}$ & This study \\
\hline P-S-PT7-sfGFP-R & $\begin{array}{l}\text { GACTGCAGCGGCCGCTACTAGTATCATTACTTATACAGCTC } \\
\text { GTCC }\end{array}$ & This study \\
\hline GroES-sF & GCATGATCGCGTGATCGTCAAGC & This study \\
\hline GroES-sR & CACACCGTAGCCATCGTTGAAAA & This study \\
\hline sfGFP- sF & GGCGTAGTTCCGATTCTGGTCGA & This study \\
\hline sfGFP-sR & TCCGGATAACGCGCAAAACACTG & This study \\
\hline T7RNAP-sF & GCATGAGTCTTACGAGATGGGTG & This study \\
\hline T7RNAP-sR & GATTTCTTGCAGGAACTGGAAGG & This study \\
\hline $16 \mathrm{~S}-\mathrm{sF}$ & GTTAAGTCCCGCAACGAGCGCAA & This study \\
\hline 16S-sR & CTTTATGAGGTCCGCTTGCTCTC & This study \\
\hline
\end{tabular}

Underlined indicated the restriction sites. 